\title{
Critique phénoménologiQue D'UNE APPROCHE NEURONALE DE LA CONSCIENCE
}

Jean-Luc Petit ${ }^{1}$

\begin{abstract}
RÉsUMÉ: La conscience est toujours conscience de quelque chose, généralement une chose autre qu'elle-même - mais quelle sorte de chose est donc la conscience, considérée en et pour elle-même? Naguère redoutable paradoxe qu'une science sérieuse abandonnait volontiers aux philosophes, la conscience a-t-elle été ramenée finalement à la condition d'un objet de science parmi les autres? Le développement d'une nouvelle "neuroscience de la conscience» depuis une vingtaine d'années est souvent présenté comme une avancée naturelle pour une science forte de son succès dans l'explication des fonctions cognitives sur la base des mécanismes neuronaux du cerveau humain. Conçue, elle aussi, originairement, comme "science de la conscience», mais sur la base de l'immanence du sujet conscient à sa propre expérience vécue, la phénoménologie doit-elle réfréner son sens du paradoxe devant le projet de cette neuroscience de la conscience, pour ne pas être accusée d'irrationalisme? Faisant retour sur le dialogue Changeux-Ricœur, je relève les objections du phénoménologue à l'objectivation de notre expérience de la conscience sur la base de mécanismes corrélatifs dans le cerveau et j'examine sur l'exemple représentatif de la théorie neuronale de Changeux, Dehaene et al. dans quelle mesure cette critique phénoménologique garde une pertinence.
\end{abstract}

Mots-CLÉs: Conscience. Cerveau. Neuroscience cognitive. Immanence. Transcendance.

\section{LA CONSCIENCE: APPROCHE EXPÉRIENCIELLE OU APPROCHE OBJECTIVE?}

Dans cet article je reviens à nouveau sur «le dialogue manqué Changeux-Ricœur», non, cette fois, dans l'intention de signaler une issue possible en spéculant sur la convergence entre la première philosophie de la volonté de Ricœur, antérieure à son tournant linguistique, et un développement prometteur des neurosciences en direction d'une physiologie de l'anticipation et de l'action (PETIT, 2006). Ce développement me semblait annonciateur d'un futur dépassement par les neurosciences du solipsisme

\footnotetext{
${ }^{1}$ Professeur à la Faculté de Philosophie (CREPHAC), Université de Strasbourg, Strasbourg - France. E-mail : jean-luc.petit4@wanadoo.fr

http://dx.doi.org/10.1590/0101-3173.2018.v41esp.06.p75
}

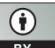

This is an open-access article distributed under the terms of the Creative Commons Attribution License. 
cérébral de la théorie de l'esprit des sciences cognitives, qui privilégient la représentation mentale au détriment de l'action, comme rapport de l'agent au monde et à autrui (BERTHOZ;PETIT, 2006, 2008). Or, sans qu'on puisse parler d'un démenti flagrant, vu que désormais il existe bien une branche des neurosciences cognitives «de l'anticipation et de l'action", cette expectative n'a, toutefois, pas été confirmée autant que je le souhaitais par la dernière décennie de travaux. D'une part, en effet, le dépassement du paradigme représentationaliste demeure ambivalent, tant que l'on reste persuadé, comme le sont les chercheurs dans ce secteur particulier des neurosciences, que l'ouverture d'un vivant sur le monde (et sur l'autre) se ramène en dernière analyse à une affaire de «représentations (ou modèles internes)", encore que projectives (non passives), à une affaire, éventuellement, de "cognition sociale (ou théorie de l'esprit d'autrui)", dont il reste vrai de dire que tout cela se passe «dans le cerveau», puisque l'argument: «où voulez-vous donc que cela se passe?» semble imparable. D'autre part, les neurosciences cognitives n'ont nullement senti le besoin de réviser leur paradigme avant de se lancer, comme elles font dernièrement, à la conquête du territoire des sciences de l'homme et disciplines annexes, sans prêter attention au fossé qu'elles devaient franchir, pour ce faire, entre l'immanence des événements mentaux ou cérébraux et la transcendance par rapport à l'activité mentale de l'individu biologique des conditions et institutions sociales, horizon de l'économie, de l'éthique, du politique, voire de la gestion des entreprises (PETIT, 2017). Au lieu de cela, l'entière stratification des niveaux d'objectivation de la vie, du comportement de l'individu aux formes de régularités globales de la société, du langage, de la culture, voire même de l'économie, est brutalement rabattue sur le plan des représentations mentales de l'esprit individuel et, de là, sur celui des systèmes fonctionnels sous-jacents dans son cerveau. Dans ce double mouvement d'expansion aux limites du domaine de la culture humaine et de repli sur le cerveau on retrouvera difficilement le double mouvement d'extériorisationintériosation qui s'est accompli à travers l'histoire de la philosophie, en culminant avec l'herméneutique de la culture - l'esprit objectif - d'un côté, la phénoménologie de la conscience - l'esprit subjectif - de l'autre. Parce que cette expansion reste confinée au mental ou au cérébral, ce repli sur l'intérieur est dépourvu de toute profondeur subjective: une grossière caricature de notre expérience de la transcendance du monde apparaissant dans l'immanence de la conscience.

Le scientifique ne peut pas prétendre être en droit de traiter d'un objet spécifique: la conscience, sans avoir à rendre des comptes au philosophe et, 
en même temps, faire des déclarations provocatrices sur la dissolution par la science de l'énigme de la conscience dans la tradition philosophique:

Transformer un mystère philosophique en un simple phénomène de laboratoire [...] Une fois que nous aurons clarifié comment l'acte de perception transforme certaines des informations qui frappent notre rétine en pensées conscientes, la montagne philosophique que nous nous faisons du caractère ineffable de l'expérience subjective accouchera d'une souris... de laboratoire. (DEHAENE, 2014, p. 24, 27).

En effet, de telles déclarations le ramènent sur le terrain même de la confrontation à laquelle il voulait se soustraire avec une approche expériencielle de la conscience, une alternative à son approche objectivante. Mais, la seule considération de la pluralité des modes d'approche possibles de la conscience n'est-elle pas de nature à prévenir le conflit entre eux? De quel droit la phénoménologie peut-elle donc se prévaloir pour critiquer une théorie scientifique de la conscience? La phénoménologie n'entre pas en compétition avec quelque science que ce soit sur le plan de la théorie, comme si elle avait en réserve une meilleure théorie de la conscience. Cela supposerait que l'objet «conscience» ait été suffisamment stabilisé en son domaine pour qu’il ne soit plus question que de trouver le modèle théorique qui en rendrait le mieux compte. Thème épistémologique récurrent dans la tradition philosophique de Descartes à Husserl en passant par Kant et Hegel, la conscience n'a que tardivement été conçue comme faculté mentale relevant d'une science empirique. De sa longue histoire pré-psychologique la phénoménologie de la conscience retient l'héritage. Tandis qu'une compétition entre approches théoriques de la conscience comme objet suppose, précisément, des théoriciens concurrents identiquement engagés dans la voie de l'objectivation. Des théoriciens qui ont quitté le sol originaire de l'expérience vécue pour adopter à l'égard de la conscience la posture de détachement, de prise de distance, de surplomb, qui est celle de l'observateur à l'égard de l'objet quelconque. À l'inverse, tout l'effort du phénoménologue vise à recouvrer sous la conscience objectivée des théoriciens la conscience comme acte, un acte personnellement accompli à travers la répétition de l'expérience de pensée fondatrice de Descartes - et la répétition de sa réactualisation par Husserl. En un mouvement contraire à celui de repousser là-devant hors de soi l'objet de considération théorique, on réeffectue l'acte de conscience de façon à décrire l'apparaitre du monde strictement du point de vue du sujet conscient. 
Le nivellement de l'objet conscience par rapport à tout autre objet de considération factuelle possible ne doit pas faire négliger le contraste majeur entre les deux attitudes: d'un côté la certitude où était Descartes et où Husserl s'est hérö̈quement maintenu face à la psychologie empirique naissante (voir ci-après) - de pouvoir procéder frontalement à une description immanente de l'expérience consciente; de l'autre la disposition permanente des psychologues à contourner le domaine de l'expérience consciente tout entier et à toujours préférer aborder la conscience par l'examen de processus mentaux inconscients, ou au seuil de la conscience, pourvu qu'ils puissent les manipuler à volonté pour les faire franchir, ou ne pas franchir ce seuil:

Gardons par conséquent le regard fixé sur le plan de la conscience et étudions ce qui se trouve contenu dans ce plan à titre immanent [...] Nous poursuivrons cette étude aussi loin qu'il est nécessaire pour obtenir l'évidence à laquelle nous avons visé, à savoir que la conscience a en elle-même un être propre qui, dans son absolue spécificité éidétique, n'est pas affecté par l'exclusion phénoménologique [de l'être naturel]. Ainsi elle subsiste comme «résidu phénoménologique» et constitue une région de l'être originale par principe, et qui peut devenir en fait le champ d'application d'une nouvelle science, - bref de la phénoménologie. (HUSSERL, 1950, p. 33).

\section{Primauté du Vécu - Dualité sémantique des discours}

Aujourd'hui comme hier, le domaine intellectuel est traversé de pressions idéologiques auxquelles le penseur doit savoir qu'il dépend de lui de céder ou résister. Aujourd'hui, c'est l'entreprise du naturalisme cognitiviste à l'égard de l'esprit humain: l'intentionnalité, la référence des représentations à un objet, l'orientation des actions vers un but, l'interaction dans la communication interpersonnelle, la subordination des conduites individuelles à des valeurs communes, toutes ces dimensions de l'expérience dont la phénoménologie avait défendu l'irréductibilité à des causalités factuelles de l'être objectif, sont ramenées à des systèmes mentaux du cerveau humain, produit de l'évolution darwinienne. Mais, qui voudra participer à un jeu dont le meneur distribuerait les cartes à son avantage, s'attribuant toujours celle du gagnant, au protagoniste celle du perdant? Or, à quoi revient le dialogue avec le scientifique quand la biologie, se voulant science cognitive, part «à la conquête de l'esprit»? Sur la base de la tradition, le philosophe pourra toujours émettre ses considérations réflexives sur l'esprit humain: au mieux il redira en termes approximatifs ce dont le savant est seul à posséder un savoir empiriquement fondé. En dehors 
de cette voie étroite: verbalisme ou fausseté! Dès ce moment, le dialogisme se referme comme un piège sur le philosophe du dialogue, victime de ses propres principes: sur l'infortuné Ricœur, le premier, qui s'est fait de l'accueil à la parole d'autrui une règle intangible.

Pour autant, quels que soient les présupposés cachés d'une discussion, si inégalement répartis que soient les avantages et les handicaps respectifs, il semble qu'un impératif s'impose de toutes façons: "construire un discours commun» est un objectif qu'on ne saurait récuser sans se déconsidérer partout. Sauf qu'un discours ne devient pas commun sur la base d'une perpétuelle ambiguïté. Ambiguïté que trahit le lexique de la naturalisation des concepts phénoménologiques. "Représentation»: tantôt l'acte de se représenter une chose; tantôt un objet mental dans le cerveau. "Action": tantôt l'épreuve d'objectivation d'une intériorité subjective; tantôt un mouvement corporel programmé par la représentation du but: «Intentionnalité»: tantôt l'orientation active de l'être subjectif dans le monde; tantôt le fait que les représentations dans le cerveau sont «au sujet de» l'objet qui les cause. Etc.

Ricœur est incomparable lorsqu'il faut mettre au jour la diversité réelle sous l'uniformité de façade dans un domaine d'investigation scientifique. C'est ce qu'il a fait pour les études historiques, non sans provoquer l'agacement des historiens, peu flattés de la bigarrure de leur prétendue positivité. Mais, c'est aussi ce dont il s'est également prouvé capable en un domaine qui n'a que tardivement attiré l'attention des philosophes, celui des neurosciences et sciences cognitives. Ce qu'il en dit dénote une sensibilité à l'hétérogène sous les regroupements disciplinaires en usage. Déjà, les neurosciences se désignaient au pluriel, mais devenant «cognitives», elles se satellisent des disciplines annexes: pluralisme redoublé. Or, dans la recherche, comme pratique effective, chaque mode d'approche génère son propre discours et chaque discours renvoie à sa propre ontologie. Sans que rien ne prouve a priori que ces discours, même si, en définitive, ils traitent "de la même chose», soient pour autant tous mutuellement traduisibles sans perte d'information. L'écologiste s'intéresse à la relation du vivant et de son milieu, l'éthologiste au comportement animal, le psychiatre à la personnalité et aux antécédents du patient, le psychologue aux fonctions psychologiques, le physiologiste aux systèmes du cerveau.

Bien sûr, l'exigence d'intégration de cette diversité se fait sentir. Mais, peut-elle à l'heure présente être autrement satisfaite qu'au plan de l'exposé pédagogique ou de la déclaration d'intention idéologique? Car, il n'existe rien de tel qu'un discours explicatif homogène qui puisse rendre compte, en termes 
des mécanismes moléculaires, chimiques ou électriques, de la synapse, par exemple, de tout l'étagement des niveaux de complexité qu'on peut distinguer entre cette synapse et le patron d'activation transitoire d'une assemblée cellulaire, ou la carte somatotopique corticale, ou la variation locale du flux sanguin cérébral, ou encore la dynamique de l'activité électrophysiologique globale. Et encore, cette énumération ne sort-t-elle pas de la région "cerveau». La démonstration devient triviale quand on passe au comportement, qui plus est au comportement dans ses conditions naturelles «écologiques». Le fait qu'on peut sauter d'un «niveau de description» au niveau inférieur en retombant à chaque fois sur "des mécanismes» n'explique pas grand-chose, vu l'amphibologie sur ces «descriptions» et «mécanismes». Ce qui est mécanisme au plan mental peut-il être mis en corrélation avec ce qui est mécanisme au plan neuronal?: c'est la question qu'on se pose à nouveaux frais, à propos de chaque fonction étudiée. Rien n'interdit, au demeurant, d'élaborer sur les bases d'une position idéologique préconçue (le monisme matérialiste tiré, hier de Haeckel, dorénavant de Spinoza) un discours à sémantique homogène qui, du fait même de cette homogénéité, pourra passer pour la vitre transparente placée devant l'être. On aura alors, sinon le Grand Discours de Vérité de la science à son terme, du moins un effort en ce sens.

Un test de la clairvoyance de Ricœur est qu'il ne partage pas l'enthousiasme de beaucoup pour les performances de l'imagerie cérébrale. «Fenêtre ouverte sur le cerveau pensant qui nous fait voir l'image des états mentaux»: qui résisterait à la fascination de la métaphore visuelle dont cette trompeuse présentation est imprégnée? Précisément, l'auteur de La métaphore vive (à distinguer des métaphores usées ou mortes!) qui nous enseigne que la métaphore fait voir les choses d'une certaine façon, parfois en fait voir alors qu'il n'y en a pas. Et aussi que la façon dont la métaphore visuelle fait voir les choses, c'est la modalité du direct. Par sa seule puissance l'objet est là, sous nos yeux. Elle est métaphore objectivante par excellence. Pour nous persuader qu'il y a "des intentions» dans les aires prémotrices, il suffit désormais qu'on puisse «les observer directement» par imagerie. Devant cette dérive métaphorique on devra toujours rappeler que l'image est pour un observateur, un contemplateur ou un rêveur. Elle n'est pas "dans son cerveau», encore moins «dans le cerveau d'un autre». Sauf à rebaptiser «image» une configuration d'activité locale (localisée par un traitement statistique des enregistrements au scanner) dont on a fait l'hypothèse qu'elle est corrélative de l'accomplissement par le sujet d'une tâche, elle-même isolée du reste de son comportement de manière à garantir la sélectivité de cette correspondance fonctionnelle. Ces réserves de 
Ricœur sont exprimées dans une critique du langage, mais qu'on ne s'y trompe pas: elles vont plus loin. Elles renvoient à la controverse entre physiologistes, informaticiens et physiciens au sujet du caractère significatif ou non des foyers d'activités dans l'image et de la comparabilité ou non comparabilité des images obtenues par des techniques différentes.

\section{LA CRITIQUE PHÉNOMÉNOLOGIQUE DE L'APPROCHE NEURONALE EST-ELLE OBSOLÈTE?}

Je me propose d'examiner dans la suite de mon propos si les développements de la théorie de la conscience défendue par Changeux, Dehaene et al. n'apporteraient pas une réponse qui soit satisfaisante pour un phénoménologue aux objections formulées par Ricœur.

1) Une théorie neuronale de la conscience est-elle fatalement un discours hybride qui mélange deux langages à sémantiques hétérogènes: un langage mécaniste de l'explication causale en termes de systèmes cérébraux, et un langage de l'expérience subjective?

Je me tiendrai, modestement mais fermement, au plan d'une sémantique des discours tenus d'une part sur le corps et le cerveau, d'autre part sur ce que, pour faire bref, j'appellerai le mental, avec les réserves que me fournissent les philosophies réflexive, phénoménologique, herméneutique. Ma thèse initiale est que les discours tenus d'un côté et de l'autre relèvent de deux perspectives hétérogènes, c'est-à-dire non réductibles l'une à l'autre et non dérivables l'une de l'autre. Dans un discours il est question de neurones, de connexions neuronales, de système neuronal, dans l'autre on parle de connaissance, d'action, de sentiment, c'est-à-dire d'actes ou d'états caractérisés par des intentions, des motivations, des valeurs. Je combattrai donc ce que j'appellerai désormais un amalgame sémantique, et que je vois résumé dans la formule, digne d'un oxymore, «Le cerveau pense». (CHANGEUX; RICEUR, 1998, p. 25).

On peut, bien sûr, soutenir qu'en dehors de quelques cas d'abus de langage sans conséquences, une théorie neuronale de la conscience peut parfaitement sauvegarder l'homogénéité sémantique requise d'une théorie explicative, à condition toutefois qu'on n'y accepte que des concepts construits sur la base des structures anatomiques et des fonctions cognitives. Fût-ce au prix d'un renvoi indéfini de la référence à l'expérience vécue du sujet conscient - ce qui devrait tout de même faire problème pour une théorie «de la conscience»! 
En ce contexte, la reprise de la thèse classique de la modularité de l'esprit (FODOR, 1983) offre une garantie d'homogénéité sémantique, puisqu'elle mobilise une conceptualité indéniablement mécaniste. En revanche, comme elle n'accorde aucun rôle à la conscience, son emploi rend problématique la réintégration dans le modèle sans contradiction de la part qui doit revenir à la conscience. De sorte qu'il va falloir regarder de près à l'articulation au sein d'une même théorie des systèmes modulaires, qui se passent du concours de la conscience, et de l'espace de travail global, ou espace de travail conscient, ou «espace conscient» avec lequel la conscience tend à être assimilée, d'autant qu'elle est évacuée des étages inférieurs du modèle théorique. Notamment, s’il est vrai que l'attention filtre l'accès à la conscience, il faudra assumer l'existence d'un système spécialisé pour l'orientation de l'attention, un système dont le caractère modulaire mettra en question son appartenance à l'espace de travail global en dépit du fait qu'il en sélectionne le contenu.

Sous l'emprise du paradigme néophrénologique de l'esprit modulaire, on ne peut probablement faire mieux que nommer "conscience» le moment oû un contenu mental accède à l'espace de travail global. C'est, en tout cas, ce que suggère la formulation prudente de Dehaene, Kerzberg et Changeux (1998), comme quoi la mobilisation de l'espace de travail global «pourrait être associée» (might be) avec un sentiment subjectif d'effort conscient. Si l'on voulait aller plus loin que cette association purement nominale, il faudrait déterminer la fonction que vient remplir la conscience. Or, on ne peut pas assigner à la conscience une fonction précise sans se voir contraint d'assumer l'existence d'un module spécial pour l'exercice de cette fonction, en contradiction avec la thèse du caractère inconscient des processus modulaires et du caractère non modulaire des opérations conscientes. Une contrainte du même ordre se fait sentir à travers la tension entre une interprétation dynamique et distributionnelle du pattern d'activité neuronale codant un contenu conscient et une tendance à confier à une aire privilégiée comme le cortex préfrontal (ou le cortex pariétal inférieur) le rôle de contrôleur du fonctionnement des systèmes modulaires subordonnés. Entre l'exigence de spécialisation anatomique ou fonctionnelle qui renvoie à des processus inconscients et la globalité de l'espace de travail conscient, on note donc un conflit irrésolu.

Le dualisme sémantique - qui se défend d'impliquer un dualisme ontologique - est surtout pour Ricœur une parade aux prétentions hégémoniques des neurosciences à l'égard de l'expérience subjective. «Le 
cerveau pense», "l'aire préfrontale prend des décisions», «l'hippocampe mémorise la trajectoire de l'organisme dans l'espace», etc.: sous prétexte d'inférer une fonction cognitive sur la base d'un mécanisme cérébral, toutes les formules de ce type trahissent un même procédé de construction par recollement sémantique de termes hétérogènes. D'un côté, le lexique de l'anatomie, ou anatomie fonctionnelle, pour décrire un objet dont les processus internes restent inaccessibles à notre expérience. De l'autre, le lexique des actes de conscience dont nous pouvons nous procurer l'expérience directe en les accomplissant. La clarification apportée par l'interdiction du mélange des lexiques n'est-elle pas compensée par un conservatisme lexical à la Gilbert Ryle? Loin de s'en tenir au constat du clivage ci-dessus, les neurosciences cognitives ont entrepris de franchir l'abîme entre les neurones et la conscience en déterminant toute une hiérarchie de niveaux d'intégration de l'information cognitive intermédiaires entre ces extrêmes. Le problème est que l'ascension de niveau en niveau ne dépend pas, ou pas uniquement, de la conscience et que l'idée d'organisation hiérarchique nous laisse démunis pour comprendre le mode d'intervention de la conscience puisqu'elle intervient seulement au sommet de la hiérarchie.

De son côté, la psychologie cognitive a tenté de ménager au témoignage du sujet sur son expérience une place qu'elle lui avait d'abord refusée. Sans doute, la reconnaissance des données de l'introspection comme "faits bruts», sans privilège par rapport aux données d'enregistrement ou d'imagerie, estelle fort loin d'accorder au sujet une intuition d'essence des structures de la conscience à la Husserl. Pour autant, on aurait sans doute tort de sous-estimer l'accueil fait au compte-rendu verbal d'introspection comme critère d'accès à la conscience. La concession ainsi faite à la conscience en termes de valeur cognitive va plus loin que la simple détection de signal, laquelle n'implique pas nécessairement un traitement d'information conscient. Le compte-rendu verbal s'avère capable de couvrir la zone grise intermédiaire entre la pleine lumière du conscient et l'obscurité complète des processus inconscients:

Des stimuli brefs ne causent qu'une activation faible, transitoire et variable de l'espace de travail. Il est tentant de voir une telle décharge transitoire comme une base neuronale potentielle pour la phénoménologie complexe des paradigmes de masquage. Aux durées d'amorçage intermédiaires (40$50 \mathrm{~ms}$ ), les sujets ne caractérisent jamais leur perception de l'amorçage masqué comme "consciente», mais ils sont nombreux à rapporter qu'ils font l'expérience occasionnelle d'entrevoir un stimulus qui semble leur échapper aussitôt, les laissant incapables de décrire ce qu'ils ont vu. (DEHAENE; NACCACHE, 2001, p. 19). 
C'est pour rendre justice à une telle extension du domaine de l'introspection aux marges de la conscience que les chercheurs ont proposé une réhabilitation du concept de "préconscient", auparavant sulfureux en sciences cognitives (DEHAENE et al., 2006). Il reste que l'accueil fait à l'expérience par le truchement du rapport verbal des sujets ne concerne qu'une expérience de sujets déguisés en observateurs scientifiques de leur propre intérieur, un intérieur qu'ils conçoivent, sur le modèle suggéré par l'expérimentateur, comme lieu d'occurence d'événements du type stimulus. Cette expérience préformatée de laboratoire semble particulièrement impropre à restituer la phénoménalité originaire de notre être-au-monde dans sa dimension paradoxale d'intériorité ouverte sur son extérieur. Un phénoménologue se gardera d'accorder trop vite l'assimilation de la conscience avec les événements ou objets mentaux auxquels cette introspection sous surveillance peut seulement donner accès.

2) Un psychique très construit...

Mais vous n'avez là qu'un psychique de laboratoire de psychologie qui n'est peut-être pas le psychique riche de l'expérience intégrale. L'être au monde est d'abord global... c'est un psychique très construit que vous mettez en relation avec un neuronal légitimement construit... Vous procédez du simple au complexe tandis que le psychique que vous mettez en relation avec le substrat neuronal est peut-être justement un psychique très, très simplifié afin de vous mettre en situation de bien le corréler avec l'architecture neuronale. (CHANGEUX; RICEUR, 1998, p. 89).

Pour illustrer ce caractère construit, on citera les protocoles expérimentaux de Dehaene, par exemple le «masquage subliminal» pour déterminer la frontière entre "voir» et «ne pas voir»: présenter une image brièvement et l'intercaler entre deux images-masques qui empêchent de la voir (DEHAENE, 2014, p. 66). Est-il besoin d'insister sur le fait que des manipulations de ce genre ne donnent accès qu'à un concept de conscience extrêmement appauvri dont la contribution à une théorie de la conscience est, du coup, problématique? Est-ce que le caractère "global» de l'espace de travail global peut tenir lieu d'équivalent dans le modèle neuronal du caractère global de la conscience? La globalité de l'espace de travail a d'abord une signification anatomique: les neurones qui le composent doivent avoir des projections axonales à longue distance vers des aires du cortex cérébral distinctes de celles qui contiennent leurs corps cellulaires. Sans doute plus pertinent est le fait qu'un contenu mental quelconque est censé pouvoir accéder à l'espace de travail pourvu que sa signature neuronale ait la saillance 
et la persistance requises. Mais, si l'on veut un équivalent encore moins approximatif de la globalité de la conscience, c'est au partage de l'information de ce contenu mental entre les modules mentaux spécialisés dans le traitement computationnel que l'on devra se référer. Autant dire que la globalité de la conscience n'a pas de représentation possible dans ce modèle, sauf à tolérer son découpage et sa répartition entre modules existants dans le système: soit le remplacement du global par le local.

3) Représentation:

La critique que la phénoménologie fait de la notion de «représentation», notion que scientifiques et philosophes tiennent trop facilement pour acquise... ce qui est mis en question, c'est l'idée d'une réplique mentale, dans l'esprit, d'une réalité extérieure, relevant d'un monde tout fait. Autrement dit, l'idée mentale considérée comme tableau réel peint «dans» la conscience fait problème. (CHANGEUX; RICEUR, 1998, p.183).

Cette critique de la représentation comme présupposant un monde organisé indépendamment de l'activité de l'organisme avait déjà eu une réponse dans l'article précurseur de Changeux et Dehaene (1989, p. 22-25). Les auteurs s'y montraient sensibles au fait de la polysémie du mot «représentation». Ils rappelaient qu'en sciences cognitives il renvoie à la fois à la structure syntaxique et au contenu sémantique d'une information internalisée (FODOR, 1975); tandis qu'en neuroscience il réfère aux projections des organes des sens sur certaines aires du cerveau ou aux projections d'un domaine cérébral sur un autre. Comme pour prévenir l'idée naïve de l'impression passive d'une image dans le tissu cérébral, il citaient deux conceptions alternatives de l'association mental-cérébral, autrement dit, du codage neuronal des représentations mentales: la conception de Hebb (1949) d'une assemblée cellulaire dont l'activité coordonnée persiste après la stimulation sensorielle et celle de Barlow (1972) qui attribue à l'activité d'une cellule unique, dite «grandmère» ou "pontificale» le codage d'une représentation. Mais du point de vue philosophique, on pourra observer que les neurophysiologistes s'intéressent surtout à l'extension, la topographie ou la corrélation temporelle de l'activité de la population neuronale responsable du codage des représentations. Tandis que le statut sémantique desdites représentations par rapport à la chose représentée demeure largement ininterrogé, ce qui justifie tous les soupçons quant à l'héritage du préjugé de l'image-copie en neuroscience. 
Toutefois, l'hypothèse du "darwinisme mental», qui fait appel aux deux concepts complémentaires: un générateur de diversité, consistant en variations spontanées d'activités oscillatoires des neurones, et une sélection a posteriori des représentations adéquates à la situation sous la contrainte de l'environnement externe, mais aussi interne, cette hypothèse pourrait être de nature à en finir avec le préjugé de l'image-copie en fondant la possibilité d'une production purement interne de représentations mentales. Ceci, dans la mesure où ce modèle neuronal de la genèse des représentations mentales repose uniquement sur la dynamique de l'activité cérébrale et ne laisse au contexte extérieur qu'un rôle marginal de contrainte de sélection. À chaque instant, de la configuration des activités synchronisées de groupes de neurones émergent des représentations, ou plutôt des "pré-représentations», lesquelles en fonction du critère de leur sélection donneront des images, des concepts, des intentions (sélectionnés sur la base de la mémoire) ou des perceptions (sur la base de la stimulation sensorielle). Pour le phénoménologue, toutefois, si le préjugé d'un monde représenté préconstitué à la représentation mentale est effectivement tenu en lisière par cette manœuvre darwinienne, son usage n'en trahit pas moins un excès de confiance du théoricien en sa capacité de recouvrer, par le détour de l'élimination du non-sens dans cette variété issue du hasard, la signification originelle de notre expérience de nous représenter un objet ou une situation.

4) Intentionnalité: Je citerai encore Ricœur sur la conception phénoménologique de l'intentionnalité, un mode de rapport du sujet au monde dans lequel le sujet ne s'éprouve pas comme enfermé dans son intérieur et uniquement occupé avec ses états mentaux, mais plutôt comme se projetant vers ses objets d'intérêt dans un contexte de situation englobant:

La conscience n'est pas une boîte dans laquelle il y aurait des objets. La notion de contenu psychique est justement un construit par rapport à l'expérience d'être dirigé vers le monde et donc d'être hors de soi dans l'intentionnalité. Je suis au monde dans un rapport très particulier, celui d'être né dans ce monde, d'être en situation. La grande avancée de la phénoménologie a été de refuser le rapport contenant/contenu qui faisait du psychisme un lieu. (CHANGEUX; RICEUR, 1998, p.137).

L'approche de l'intentionnalité du point de vue de ses bases neurales conduit à substituer à ce qui n'est justement pas un objet, mais plutôt un mode subjectif d'être en rapport à..., un certain type d'objet mental, nommé «intention», que caractérise son occurrence à un haut niveau d'organisation 
(le cortex préfrontal où se déterminent les buts de l'action et d'où s'exerce le contrôle sur sa réalisation). Une telle substitution passe inaperçue d'autant que l'intérêt des chercheurs se focalise sur le mécanisme neuronal susceptible d'assurer l'implémentation de ce type d'objets dans le cerveau. Que l'on puisse assigner à l'intentionnalité une configuration d'activité neurale simplement caractérisée par le fait qu'elle se produit spontanément à un certain niveau d'organisation et qu'elle n'est pas contrainte par l'état actuel de l'environnement, cela semble une évidence qui dispense le neurophysiologiste de la tâche d'une analyse phénoménologique de l'expérience intentionnelle. Le collapse d'un mode d'être subjectif sur un objet mental et de cet objet mental sur un état d'activité neuronale est un geste si naturel à la recherche des corrélats neuronaux que sa légitimité ne fait pas question. À tel point que «l'intention» - objet mental entièrement reconstitué sur la base de l'activité neuronale corrélative - occupe la place de l'irréductible intentionnalité: une opération indissociable du processus d'objectivation lui-même, et dont il est vain de chercher la trace dans ce que le théoricien a la rassurante modestie de ne proposer que comme un modèle neuronal.

5) Introspection ou intersubjectivité:

Une composante verbale, déclarative, est immédiatement incluse dans les procédures d'expérience. L'expérimentateur ne peut éviter de faire crédit à ces rapports, quitte à les contrôler par d'autres... Le langage nous fait sortir de la subjectivité privée. Le langage est un échange qui repose sur plusieurs présuppositions. D'abord, la certitude que les autres pensent comme je pense, voient et entendent comme moi, agissent et souffrent comme moi. Ensuite, la certitude que ces expériences subjectives sont à la fois insubstituables (vous ne pouvez pas vous mettre à ma place) et communicables (essayez, je vous prie, de me comprendre!)... Il y a une herméneutique de la vie quotidienne qui donne à la prétendue introspection la dimension d'une pratique interpersonnelle. (CHANGEUX; RICCEUR, 1998, p. 83).

Dans la phénoménologie intuitionniste de Husserl la conscience me procure une ouverture sur un monde où je ne suis pas seul, dans la mesure où parmi les choses que j'y perçois l'Einfühlung m'apprésente certains corps qui sont d'autres corps propres comme mon propre corps propre. La possibilité d'un tel accès perceptif à la vie intérieure d'autrui témoigne peut-être du fait que ma conscience n'est pas solitaire, mais celle-ci n'en reste pas moins originairement mienne, puisque l'inscription d'autrui dans mon environnement dépend de 
l'extension par l'empathie de ma perception. Or, la précédence du moi comme sujet de la perception fait paradoxe avec la doctrine phénoménologique de l'intersubjectivité première en soi par rapport à ma subjectivité personnelle. La phénoménologie herméneutique de Ricœur a heureusement remédié à ce paradoxe (PETIT, 2011). Si je suis plus fondamentalement sujet parlant que sujet percevant, la précédence du «comme un autre» par rapport à l'être identique à soi-même ne fait que résumer la profondeur du champ herméneutique de ma conscience langagière. L'autre ne se réduit pas à un corps apparié avec le mien comme un autre corps propre. M'adressant à quelqu'un comme capable de me comprendre, je ne le promeus pas seulement comme un autre centre de perspective sur le monde, mais je lui reconnais d'avance - et sans avoir recours à un jugement attributif d'états mentaux le statut de co-participant au monde de l'esprit, incluant la variété des états et actes de conscience exprimables. De cette profondeur intersubjective de l'horizon herméneutique de la communication, le critère des neurosciences cognitives de «la reportabilité d'un état de conscience» garde fort peu. Certes, la garantie d'accès du contenu d'information cognitive correspondant à l'espace de travail conscient que procure le fait de pouvoir être rapporté dans un énoncé verbal est sans réserve: le sujet ne peut manquer d'avoir conscience du percept, du souvenir ou de l'image qu'il dit avoir ou éprouver. En revanche, la minceur de cette capacité de faire un rapport verbal de l'occurrence d'un état ou événement interne n'a pas à être soulignée. Comme usage du langage, comme mode d'interaction symbolique, comme relation intersubjective $-\mathrm{y}$ compris dans le contexte immédiat du laboratoire - cette capacité cognitive semble s'ignorer elle-même. Le caractère hautement artificiel de la posture présumée être celle du sujet, comme spectateur-et-rapporteur d'états de choses strictement privés, passe complètement inaperçu dans le milieu des chercheurs en sciences cognitives. De sorte que rien ne vient faire obstacle au collapse de ce rapport verbal de représentation consciente sur son corrélat neuronal dans le modèle de l'espace de travail conscient, à savoir une étape avancée, voire préterminale, du traitement de l'information cognitive, où les aires frontotemporales du langage étant sélectivement activées, l'entrée en jeu du système de contrôle préfrontal est irréversible, de même que la diffusion à distance de l'information traitée sur l'axe fronto-pariétal pour son relais vers d'autres processeurs cérébraux, etc.

6) Le précepte de ne pas mutiler «le champ phénoménologique large» de l'expérience intégrale des hommes (CHANGEUX; RICCEUR, 1998, p. 195) fait appel à la phénoménologie pour une restitution d'amplitude à ce champ 
qu'on est obligé de réduire pour "faire de la psychologie» (CHANGEUX; RICEEUR, 1998, p. 146). La phénoménologie accomplit à rebours le chemin de l'objectivation scientifique en ses trois étapes: 1) le détachement de l'objet mental du vécu d'un sujet; 2) le détachement de cet objet de l'horizon du monde de vie de ce sujet; 3) l'inclusion de cet objet détaché à l'intérieur d'un réseau d'objets également détachés: un système neuronal. Ce processus d'objectivation, il n'est plus question de l'interdire, comme voudrait «une phénoménologie intransigeante» (CHANGEUX; RICCEUR, 1998, p.143) repliée sur une subjectivation abusive qui «remet l'intentionnel dans la conscience» (une allusion probable au Husserl des Ideen I). «Expliquer plus pour comprendre mieux»: la maxime de Ricœur contient l'esquisse d'une "coordination entre compréhension (vécue) et explication (objective)». Cette coordination consiste à «montrer que la possibilité d'objectiver est comprise dans la relation intentionnelle, dans la relation de signifiance, et dans la mise en commun du sens à plusieurs", soit une conception enrichie de l'intentionnalité qui permet au phénoménologue-herméneute de reconduire l'objet mental ou ses corrélats cérébraux à la conscience, telle qu'on l'entend "en usant seulement de la vie et des conversations ordinaires" (DESCARTES, [1643] 1989). Sortant du laboratoire de psychologie pour nous replonger dans le monde de la vie quotidienne, nous retrouvons le contexte originel où nous avons appris ce que «conscience» veut dire. Chacun sait distinguer la bonne conscience de la mauvaise, la conscience tranquille des remords de conscience; des professionnels on exige qu'ils fassent preuve de conscience professionnelle; on s'engage dans l'action politique suite à une prise de conscience de l'injustice de l'ordre établi, etc.: il est remarquable que l'on ne renvoie pour ainsi dire jamais à une condition psychologique de l'individu isolé, mais toujours à une disposition pratique ou morale de l'agent en situation d'interaction sociale. L'expliquer dépouille l'état de conscience de ses dimensions pratiques ou, plus généralement, interactionnelles, sous lesquelles nous le comprenons normalement. Faisant à rebours le long chemin de l'objectivation de la conscience en repassant par les étapes successives de sa réduction aux états ou événements de la matière cérébrale, nous accédons à une intelligibilité supérieure en ce que le phénomène non réduit se voit rétabli en sa priorité naturelle. Autant l'extraction hors contexte suscite l'exigence d'explication, autant la réinsertion en contexte ramène sur le sol primordial du vécu inquestionné, parce que préalable à toute mise en question.

Mais si une telle régression vers les contextes d'usage originaires des concepts de conscience peut bien apaiser, sinon satisfaire la demande 
d'explication, il reste douteux qu'elle suffise à déterminer les caractéristiques spécifiques de la conscience. Démarche de recontextualisation, celleci évoquerait plutôt un relativisme contextualiste à la Wittgenstein, qui désubstantialise l'objet de référence des substantifs et disperse la référence sur la variété infinie des occasions d'usage des mots et autres pratiques interactionnelles. Le risque encouru par là est sans doute inévitable, dès lors qu'on a perdu la confiance de Husserl et des phénoménologues réalistes de première génération (Adolph Reinach) en la possibilité d'une éidétique fondée sur l'intuition d'essence du sujet des actes de conscience. L'interprétation des expressions verbales (ou gestuelles) de l'être-conscient, que Ricœur préconise comme solution de rechange, est un excellent palliatif au défaut d'une phénoménologie purement descriptive et intuitive, mais il a son prix, qui est de disperser la référence sur la variété des usages des mots en se reposant sur la vertu clarificatrice de la conversation.

Les neurosciences sont-elles mieux armées pour capturer les caractéristiques spécifiques - une science empirique ne vise pas les caractéristiques essentielles - de la conscience? Voyons plutôt. On peut entendre le «détachement de l'objet mental» qu'est une représentation consciente d'avec son contexte d'expérience vécue comme renvoyant à l'option méthodologique des neurosciences de la conscience. Celles-ci ont tenté d'échapper à l'enfermement dans l'immanence à soi-même du vécu de conscience grâce au paradigme de masquage subliminal, qui est conçu pour révéler l'existence d'une activité d'encodage et de traitement cognitif du stimulus indépendante de la conscience. Ce complet contournement du champ phénoménologique de la conscience présente un avantage évident et un inconvénient qui l'est peut-être moins. L'avantage est de pouvoir explorer la limite de la conscience en manipulant la visibilité du stimulus tout en sauvegardant son impact sur le système. L'inconvénient est que l'affinement de ce même paradigme de masquage subliminal tend à une extension illimitée du domaine des processus cognitifs inconscients, plutôt qu'à une délimitation plus stricte d'un domaine propre de processus conscients. Au moins, la contribution de l'étude du traitement inconscient de l'information cognitive à cerner les bases de la conscience est-elle plus ambivalente que ne l'admettent les chercheurs (DEHAENE; NACCACHE, 2001). De sorte que le refus d'une approche immanente et réflexive des caractéristiques de la conscience en faveur d'une approche expérimentale (avec ce paradigme) expose à ce qu'aucune des caractéristiques retenues dans le modèle de la conscience n'échappe à l'éventualité de se voir démentie par une version plus sophistiquée 
du paradigme, qui démontre la compatibilité de cette caractéristique avec une activité mentale inconsciente.

Dans le modèle hiérarchique combinant un étage inférieur de processeurs modulaires subordonnés et un étage supérieur caractérisé comme espace de travail non modulaire pour le partage de l'information cognitive entre processeurs et le contrôle des stratégies cognitives, la programmation des tâches et la communication d'information à distance sont des fonctions de contrôle de haut niveau qu'il est tentant de réserver à la conscience. Pour autant, le fait que ces mêmes fonctions recrutent des réseaux neuronaux susceptibles d'être activés par des stimuli inconscients a été établi par des travaux en imagerie cérébrale et en électroencéphalographie.

Dans une recherche en imagerie fMRI (LAU; PASSINGHAM, 2007), les sujets doivent réagir à la présentation de mots par un jugement, qui peut être d'ordre phonologique: «dissyllabique?», ou d'ordre sémantique: «concret ou abstrait?». On sait que ces deux types de jugements recrutent des régions corticales distinctes: un jugement phonologique implique le cortex prémoteur ventral gauche; tandis qu'un jugement sémantique intéresse le gyrus temporal moyen gauche ainsi que le cortex préfrontal inférieur gauche. L'instruction relative à la tâche commandée est précédée d'un indice d'amorçage qui peut être subliminal et préparer la tâche alternative. Quand la tâche alternative est amorcée inconsciemment, l'image fMRI montre une baisse d'activité dans les aires mobilisées par la tâche explicitement commandée et un accroissement d'activité dans les aires mobilisées par la tâche alternative. Une preuve du fait qu'il suffit d'un indice inconscient pour induire l'engagement du système cognitif dans la tâche alternative par rapport à celle de la commande explicite. L'activation corrélative du cortex préfrontal dorsolatéral, base présumée du contrôle cognitif, démontre l'existence d'une influence inconsciente sur le système de contrôle cognitif jusque dans la programmation de tâches complexes. Une réfutation de l'idée que la fonction de contrôle cognitif de haut niveau requiert la conscience.

Dans une recherche presque contemporaine en électroencéphalographie (COHEN et al., 2009), un signal visuel de commande d'une réponse pressebouton est précédé d'un indice d'inhibition d'action de façon à induire une erreur. Erreur dont les sujets ont conscience lorsque cet indice d'inhibition est visible, mais qui peut aussi passer inaperçue lorsqu'il est rendu inconscient par masquage. Les enregistrements EEG au niveau des cortex préfrontal médian et occipital lors des essais sans erreur succédant à des essais avec erreur, permettent 
l'examen de la connectivité fonctionnelle entre ces régions extrêmes du contrôle cognitif sur la perception visuelle. Mesure de la connectivité fonctionnelle, la synchronisation de phase des oscillations électriques cérébrales du cortex préfrontal médian et du cortex occipital est renforcée de façon significative à la suite d'erreurs, même dans le cas où les sujets ne sont pas conscients de ces erreurs. Preuve qu'une information inconsciente est tout à fait capable d'activer un processus de contrôle centrifuge sur une région sensorielle périphérique pour une correction d'erreur, lors même que le sujet percevant ignore l'avoir commise. Ce résultat suggère, contrairement à l'hypothèse de Dehaene et Changeux, que la synchronisation à distance préfrontale-occipitale n'est pas une caractéristique unique de la conscience. On ne se bercera pas d'illusions en affirmant que ces contre-exemples suffisent à réfuter le modèle d'espace de travail conscient, vu que ce modèle est trop éclectique et même consensuel dans sa composition pour être rigoureusement falsifiable. Du propre aveu des auteurs (DEHAENE; NACCACHE, 2001, 4.2.), en effet, leur modèle «fait la synthèse des idées» des nombreux et influents chercheurs qui complètent le modèle - partiellement vrai, donc - d'esprit modulaire en introduisant un mode de traitement cognitif non modulaire: Posner (1994), Baddeley (1986), Shallice (1988), Baars (1989), Tononi et Edelman (1998).

En adaptant le critère de Haynes (2009), on pourra dire que l'activation du cortex préfrontal ou la synchronisation avec l'activité électrique du même cortex est peut-être un corrélat neuronal de la conscience, celà ne suffit pas pour annoncer la découverte du "code de la conscience». Un code doit permettre non seulement l'encodage d'une dimension particulière de l'expérience dans un pattern d'activité cérébrale corrélative, mais surtout le décodage univoque de cette dimension de l'expérience sur la base de cette activité cérébrale. Or, en l'occurrence, cette condition n'est pas remplie, puisque la même configuration d'activité cérébrale est enregistrée à la fois lors d'un processus de contrôle de tâches accompagné et non accompagné de conscience. D'un point de vue phénoménologique, on pourrait avancer l'idée que ce qui manque à une recherche des corrélats de la conscience dominée unilatéralement par un modèle de connectivité cérébrale pour s'assurer de l'univocité d'un code de la conscience, c'est une analyse parallèlement conduite de l'expérience vécue sur le terrain propre de cette expérience. De fait, les caractéristiques du traitement conscient de l'information cognitive retenues comme possiblement spécifiques dans le modèle d'espace de travail global sont induites à partir de l'architecture fonctionnelle neuronale postulée par le modèle. Contrôle central des stratégies cognitives, sérialité du traitement de l'information, 
fluctuations spontanées indépendantes du stimulus, accessibilité globale de l'information, stabilisation des représentations par boucles réentrantes, etc. Aux antipodes d'une description phénoménologique du champ phénoménal de la conscience, ces caractéristiques prétendues présupposent une tacite assimilation du vécu de conscience avec son hypothétique fondement cérébral. Le modèle théorique fait écran à l'expérience directe en même temps qu'il la reconstruit dans un espace de représentation orthogonal à son propre plan. La conscience, de ce fait, tend à être absorbée, plutôt qu'expliquée, par ses corrélats neuronaux. Bornant ses ambitions, côté expérience, à rendre compte des données de laboratoire obtenues avec le paradigme «vu/pas vu» (masquage subliminal, etc.), les neurosciences de la conscience tournent le dos à l'expérience sans rivage de la conscience. Un défaut rédhibitoire de spécificité des caractéristiques candidates qui est peut-être la sanction pour le refus initial de prendre position sur le terrain de la conscience et le choix d'un point de départ qui n'en est pas un en commençant par ignorer le phénomène lui-même au profit d'hypothèses sur les causalités sous-jacentes.

7) Si la critique phénoménologique n'est pas complètement inaudible en neurosciences cognitives, c'est sans doute qu'elle fait un geste en direction d'une restitution de l'expérience intégrale. L'objectivation scientifique de l'expérience subjective procède essentiellement par réduction, mais ce qui la justifie est qu'elle ne s'en tient pas à cette réduction. En admettant que l'expérience réduite nous donne les représentations mentales accédant à l'espace de travail conscient, la question est de savoir ce qu'il faut ajouter au modèle pour rejoindre l'expérience intégrale. Mais que veut dire «ajouter»? Sous prétexte de compléter la part objectivable de l'expérience, faire appel à l'ineffable n'ajoute rien. Et si l'accès à l'espace de travail conscient est bien la condition de possibilité d'un objet mental, alors on cherchera en vain dans l'expérience subjective de quoi ajouter aux représentations mentales de la psychologie cognitive. Tel est le dilemme: ou bien vous creusez un abîme entre l'expérience subjective et ce qu'en peut intégrer le modèle neuronal de la conscience; ou bien vous préjugez cette expérience d'avance conforme à sa représentation dans le modèle. L'incommensurabilité des termes dans un cas, leur homogénéité dans l'autre annule l'opération: rien à ajouter!

La phénoménalité de l'apparaître à un sujet percevant-et-agissant d'un objet, d'une situation et, plus généralement, d'un monde de vie en commun avec les autres, est ce dont Ricœur entendait rappeler la précédence par rapport à toutes les objectivations scientifiques de notre expérience en termes d'objets mentaux 
et de leurs corrélats cérébraux. Sans doute ce rappel était-il trop réminiscent de Heidegger pour être audible en neurosciences cognitives, du moins pas sans un filtrage préalable par une philosophie analytique moins cognitive-incompatible. C'est donc de préférence à la distinction de Ned Block (2005) entre «conscience d'accès» et "conscience phénoménale» que Dehaene réagit (DEHAENE, 2014, p. 25-26) quand il soutient contre «certains philosophes qui continuent de prétendre que toutes les avancées scientifiques ne changent rien au cœur du problème»: «Le nœud du problème consiste à comprendre comment une information accède à la conscience». La distinction en cause était pourtant conçue en des termes plus acceptables pour le théoricien du traitement computationnel de l'information cognitive que ceux de la phénoménologie. Block se contente de faire observer qu'un "contenu d'expérience» peut être bien déterminé en sa catégorie perceptive (comme couleur ou comme mouvement perçu) sans avoir pour cela à accéder à l'espace de travail conscient. En conformité avec la doctrine cognitiviste - et non sans une notable insensibilité phénoménologique - la notion désubjectivée de «contenu d'information» (encodé pour son traitement par le système cognitif) a été substituée à l'expérience vive du sujet percevant. Or, le vécu ne se laisse pas réduire au caractère informationnellement défini (comme couleur, mouvement ou visage) d'un contenu d'expérience. Excepté lorsqu'on tolère l'amalgame: contenu = phénoménologie $=$ conscience (BLOCK, 2005, p. 48: "That content itself is a kind of phenomenology, a kind of consciousness»). Parce que l'important n'est pas la dimension expériencielle du «contenu phénoménologique», mais seulement d'établir la distinction des corrélats neuronaux respectifs de la conscience d'accès et de ladite «conscience phénoménale».

Toutefois, même dégagé de toute référence compromettante à un vécu phénoménologiquement décrit, le plaidoyer ne convainc pas, parce que la conscience phénoménale n'a tout simplement pas sa place dans le modèle d'espace de travail conscient. Pour soustraire le modèle à toute confrontation possible avec un vécu subjectif, le sérieux de la discussion épistémologique est moins efficace que d'agiter l'épouvantail de l'irrationnel:

[L]a «conscience phénoménale»: l'intuition, profondément ancrée en nous, que chacune de nos expériences conscientes est un phénomène "en soi», qu'elle possède une qualité particulière, un quale irréductible de pensée [...] Par nature, ces états internes, personnels et subjectifs, échapperaient à toute tentative de description objective [...] la notion d'une "conscience phénoménale», intrinsèquement différente de celle d'accès conscient est trompeuse et nous entraîne sur la pente glissante qui mène au dualisme. (DEHAENE, 2014, p. 26). 
Si la conscience phénoménale est purement et simplement évacuée de l'horizon d'une neuroscience de la conscience, cette éviction n'est pas sans laisser le sentiment d'un vide à combler. Difficile de l'avouer sans remettre en cause l'option méthodologique initiale de considérer non le phénomène même de la conscience, mais l'élément d'information accédant à la conscience. C'est le même geste fondamental qui se répète à tous les niveaux du traitement d'information dit conscient: quoi que veuille dire «conscient», on ne retient que le processus de traitement, sous prétexte que l'on sait décrire celui-ci, alors même qu'on ne sait rien de l'expérience subjective qui l'accompagne - et qu'on n'en saura pas plus à l'issue de ce traitement - en dépit du fait que le sens de toute la démarche est suspendu à la conviction des chercheurs qu'au bout du compte la conscience se trouvera expliquée. Expliquée par ses causes dans les états d'activation de l'architecture fonctionnelle du cerveau sans que le vécu du sujet ait du être convoqué. Mais, estil absolument satisfaisant que le processus d'explication se referme sur lui-même en détachant la structure informationnelle de la conscience d'avec son substrat vécu dans une expérience subjective? Ne laisse-t-on pas échapper une partie de l'information pertinente pour le système cognitif en négligeant la fonction qui rattache cette structure informationnelle à l'organisme qui en assure le traitement? La conscience de soi se recommande ainsi à l'attention du chercheur, si indifférent qu'il soit à l'expérience subjective. Celle-ci continue d'être ignorée dans un modèle qui accueille la conscience de soi comme «instance particulière d'accès conscient où le projecteur conscient est orienté vers des états internes» (DEHAENE et al., 2014). Strictement encadrée entre une introspection, qui n'a jamais accès à l'élaboration interne de nos processus cérébraux, et une métacognition qui représente récursivement n'importe quel contenu de représentation cognitive, cette ainsi nommée "conscience de soi» mobilise les nombreux circuits modulaires de la représentation de soi (cartes somatotopiques, circuit des visages, module de la théorie de l'esprit, etc.) dans l'espace de travail conscient pour permettre leur inspection par d'autres processus, tout aussi modulaires (DEHAENE; NACCACHE, 2001). Telle est du moins la traduction, dans une terminologie fonctionaliste barricadée contre l'expérience subjective, des bases cérébrales de la conscience réflexive. Son intégration au modèle de la conscience ne présente pas, chacun le voit, le caractère d'une révision déchirante!

\section{Conclusion}

Dans cet article j'ai tenté de caractériser le mode d'approche de la conscience par les neurosciences de la conscience considérées dans l'une des 
plus influentes théories actuelles. Qu'il y a lieu d'attirer l'attention sur le mode d'approche de la conscience par ces sciences, plutôt que sur les faits qu'elles sont supposées avoir établi concernant la conscience, comme on fait d'habitude dans la littérature de vulgarisation, c'est ce qui ressort d'une critique phénoménologique inspirée par le dialogue Changeux-Ricœur. Sans la toile de fond d'une phénoménologie de la conscience, on manquerait en effet du recul nécessaire pour se déprendre de la fascination par les faits et reconsidérer ceux-ci à partir de leur mode d'approche. Et, par la même occasion, prendre connaissance de la singularité de ce mode d'approche de la conscience par les neurosciences, en ce qu'il procède d'une attitude qui n'est pas neutre et qui ressort mieux par contraste avec le phénomène au sens de la phénoménologie: l'expérience du monde apparaissant. Chez ceux qui poursuivent le projet d'une neuroscience de la conscience, l'hypostase des objets de la connaissance scientifique et de la manipulation technologique emprunte la forme d'une conviction généralement partagée selon laquelle la conscience doit être quelque chose du cerveau. De sorte que ce qu'on connaît des mécanismes cérébraux devrait suffire à rendre compte de la manière dont le cerveau engendre la conscience. Nous ne sommes rien d'autre que des assemblées complexes de neurones et nous avons des états de conscience (DEHAENE et al., 2017): prenant fermement appui sur ce sol de certitude, on croit s'en tenir à de l'être objectif et pouvoir laisser tomber le processus subjectif préalable de l'objectivation. En vérité, ce que révèle la critique phénoménologique, c'est que ce socle ontologique est sous-tendu par un ensemble de manœuvres épistémologiques très cohérent - quoique implicite et soustrait à la discussion -, des manœuvres qui lui confèrent une apparence de stabilité uniquement due au refoulement de la confrontation directe avec le phénomène même de la conscience.

À l'intention des lecteurs qui pourraient s'estimer frustrés par mon scepticisme en ce qui concerne les perspectives de dialogue entre phénoménologie et neurosciences de la conscience, et qui m’objecteraient l'existence d'une «neuro-phénoménologie» comme preuve de la possibilité d'une hybridation des deux approches de la conscience, permettez-moi de renvoyer à mon article (PETIT, 2014). Comme contribution à l'histoire du projet de la neuro-phénoménologie d'entrelacer (d'après une interprétation charitable) la phénoménologie husserlienne de la conscience avec la neuroscience du fonctionnement cérébral en exploitant l'analogie entre la cohérence oscillatoire - base neuronale présumée d'un état de conscience - et l'Einstimmigkeit der Erfahrung-opérateur de la constitution transcendantale-, 
j'y retrace l'ouverture puis la fermeture d'une apparente fenêtre d'opportunité, tant en phénoménologie qu'en neurosciences, pour la réalisation (finalement manquée) de ce projet.

PETIT, J.-L. Crítica fenomenológica de uma abordagem neuronal da consciência. Trans/ Form/Ação, Marília, v. 41, p. 75-100, 2018. Edição Especial.

Resumo: A consciência é sempre consciência de algo, geralmente algo diferente de si - mas que tipo de coisa é a consciência, considerada em si mesma? Paradoxo a pouco tempo temido de que uma ciência séria se entrega de bom grado aos filósofos, será que a consciência foi finalmente reduzida à condição de um objeto da ciência, dentre os demais? O desenvolvimento de uma nova "neurociência da consciência" por cerca de vinte anos é frequentemente apresentado como um avanço natural para uma ciência segura de seu sucesso na explicação de funções cognitivas baseadas nos mecanismos neurais do cérebro humano. Concebida, também, originalmente como uma "ciência da consciência", mas com base na imanência do sujeito consciente à sua própria experiência vivida, a fenomenologia deve refrear seu sentido de paradoxo frente ao projeto da neurociência da consciência, para náo ser acusada de irracionalismo? Retornando ao diálogo Changeux-Ricoeur, considero a crítica da objetivação fenomenológica da nossa experiência de consciência com base em mecanismos internos ao cérebro, e examino o exemplo representativo da teoria neuronal de Changeux e Dehaene et al., para avaliar até que ponto a crítica fenomenológica permanece relevante.

Palavras-Chave: Consciência. Cérebro. Neurociência cognitiva. Imanência. Transcendência.

\section{RÉFÉRENCES}

BAARS, B. J. A cognitive theory of consciousness. Cambridge: Cambridge University Press, 1989.

BADDELEY, A. D. Working memory. Oxford: Clarendon Press, 1986.

BARLOW, H. B. Single units and sensations: a neuron doctrine for perceptual physiology? Perception, v. 1, n. 4, p. 371-394, 1972.

BERTHOZ, A.; PETIT, J.-L. Physiologie de l'action et phénoménologie. Paris: Odile Jacob, 2006/The Physiology and Phenomenology of Action. Translation by Christopher Macann. Oxford: Oxford University Press, 2008.

BLOCK, N. Two neural correlates of consciousness. Trends in Cognitive Sciences, v. 9, n. 2, p. 46-52, 2005.

CHANGEUX, J.-P.; RICEUUR, P. Ce qui nous fait penser : la nature et la règle. Paris: Odile Jacob, 1998. 
.; DEHAENE, S. Neuronal models of cognitive functions. Cognition, v. 33, n. $1 /$ 2, p. 63-109, 1989.

COHEN, M.; et al. Unconscious errors enhance prefrontal-occipital oscillatory synchrony. Frontiers in Human Neuroscience, v. 3, n. 54, p. 1-12, 2009.

DEHAENE, S. Le code de la conscience. Paris: Odile Jacob, 2014.

. et al. Conscious, preconscious, and subliminal processing: a testable taxonomy. Trends in Cognitive Sciences, v. 10, n. 5, p. 204-211, 2006.

. et al. Toward a computational theory of conscious processing. Current Opinion in Neurobiology, v. 25, p. 76-84, 2014.

.; KERSZBERG, M.; CHANGEUX, J.-P. A neuronal model of a global workspace in effortful cognitive tasks. Proc. Natl. Acad. Sci. USA, v. 95, p. 14529-14534, 1998.

.; LAU, H.; KOUIDER, S. What is consciousness, and could machines have it?, Science, v. 358, n. 6362, p. 486-492, 2017.

.; NACCACHE, L. Towards a cognitive neuroscience of consciousness: basic evidence and a workspace framework. Cognition, v. 79, n. 1/2, p. 1-37, 2001.

DESCARTES, R. Descartes à Élisabeth, lettre du 28 juin 1643. In:

Correspondence avec Élisabeth. Paris: GF-Flammarion, 1989, p. 74.

FODOR, J. The language of thought. Cambridge, MA: Harvard University Press, 1975.

. The modularity of mind. Cambridge, MA: MIT Press, 1983.

HAYNES, J.-D. Decoding visual consciousness from human brain signals. Trends in Cognitive Sciences, v. 13, n. 5, p. 194-202, 2009.

HEBB, D. O. The organization of behavior: a neuropsychological theory. New York: Wiley, 1949.

HUSSERL, E. Idées directrices pour une phénoménologie. Traduction de

Paul Ricœur. Paris: Gallimard, 1950.

LAU, H.; PASSINGHAM, R. Unconscious activation of the cognitive control system in the human prefrontal cortex. The Journal of Neuroscience, v. 27, n. 21, p. 5805-5811, 2007.

PETIT, J.-L. Sur la parole de Ricœur: le cerveau ne pense pas: je pense. Revue d'Histoire et de Philosophie Religieuses, t. 86, n. 1, p. 97-109, 2006.

. Le tournant diltheyen de la phénoménologie: Ricœur et Husserl. In: BÜHLER, P.; FREY, D. (dir.). Paul Ricœur: un philosophe lit la Bible. à l'entrecroisement des herméneutiques philosophique et biblique. Genève: Labor et Fides, 2011. p. 167-179. 
Naturalizing Husserlian phenomenology along a Leibnizian Pathway. AVANT. Trends in Interdisciplinary Studies, v. 5, n.2, p. 218-231, 2014.

Neurosciences appliquées: une question de limites. In: COLLOQUE

SIMPLEXITE ET MODELES OPERATIONNELS. Collège de France, 28-29 Juin 2016, Actes [...] Angela Minzoni et Eléonore Mounoud (éd.). Eds CNRS Alpha, 2017.

POSNER, M. I. Attention: the mechanisms of consciousness. PNAS USA, v. 91, n. 16, p. 7398-7403, 1994.

SHALLICE, T. From neuropsychology to mental structure. Cambridge: Cambridge University Press, 1988.

TONONI, G.; EDELMAN, G. M. Consciousness and complexity. Science, v. 282, n. 5395, p. 1846-1851, 1998.

Recebido: 15/11/2018

Aceito: $15 / 11 / 2018$ 
PETIT, J.-L. 